\title{
A Comparative Study of Muscle Symptoms of Atorvastatin with Rosuvastatin in Patients of Atherosclerotic Cardiovascular Disease
}

MD. RAKIBUL HASAN RASHED, SAJAL KRISHNA BANERJEE, SYED ALI AHSAN, S M MUSTAFA ZAMAN, MD. KHURSHED AHMED, MD. MUKHLESUR RAHMAN, MANZOOR MAHMOOD, JAHANARA ARZU, DIPAL KRISHNA ADHIKARY, MD. GOLAM HOSSAIN RABBANI, MD RASUL AMIN, MD. MOSTASHIRUL HAQUE, MOHAMMED RAYHAN MASUM MONDOL, MD FAKHRUL ISLAM KHALED, MD. FAISAL IBN KABIR, MD ASHRAF HOSSAIN, MD AL AMIN, SHEIKH MOHAMMAD SAMSUZZAMAN, MD MASHIUL ALAM, MD. JAINAL ABEDIN, MD. NOORNABI KHONDAKER, ATM IQBAL HASAN, HARISUL HOQUE

University Cardiac Center, Bangabandhu Sheikh Mujib Medical University, Shahbag, Dhaka, Bangladesh

Address of Correspondence: Dr. Md. Rakibul Hasan Rashed, University Cardiac Center, Bangabandhu Sheikh Mujib Medical University, Shahbag, Dhaka, Bangladesh, Email: rakibulrashed.rr.20@gmail.com

\begin{abstract}
Background: Statins are the corner stone therapy of atherosclerotic cardiovascular disease (ASCVD). Statins may cause myalgia, myotoxicity, myopathy and rhabdomyolysis along with its lipid lowering properties and pleiotropic effects. Statins associated muscle symptoms (SAMS) are the leading cause of nonadherent and discontinuation. This study was conducted to evaluate and understand the muscle symptoms of high intensity statin therapy (atorvastatin $40 \mathrm{mg}$ and rosuvastatin $20 \mathrm{mg}$ ) for a period of three months in individual patient with clinical atherosclerotic cardiovascular disease.

Methods: A total of 280 patients with clinical atherosclerotic cardiovascular disease were studied to once daily atorvastatin $40 \mathrm{mg}$ and rosuvastatin $20 \mathrm{mg}$. It was a randomized controlled single blind trial. The primary end point was muscle symptoms-muscle pain, fatigue, cramp/spasticity and weakness at 4 weeks and in 3 months of study period. Serum creatinine kinase was measured in every patient with muscular symptoms.

Results: Patients of atorvastatin group noticed severe pain more than rosuvastatin group at the end of 3 months of treatment period $(14.21 \% \mathrm{vs} 4.38 \%, p<0.05)$, respectively). Significantly more patients felt extremely bad $(12.78 \%$ vs $4.38 \%, p<0.05)$ and bad $(24.66 \%$ vs $14.52 \%, p<0.05)$ with atorvastatin compared with rosuvastatin. Patients of atorvastatin group showed more marked increase muscle spasm (3.76\% vs $1.46 \%, p<0.05)$ and slight increase muscle spasm $(36.27 \%$ vs $16.01 \%, p<0.05)$ than rosuvastatin group by spasticity grade. One patient of atorvastatin group developed considerable increase in muscle spasm. Medical research council (MRC) muscle power grade 4 between atorvastatin and rosuvastatin group was observed $20.05 \%$ vs $10.90 \%, p<0.05$, respectively. Three patients of atorvastatin group developed grade 3 muscle power. Serum creatine kinase $>1500 \mathrm{U} / \mathrm{L}$ was observed more in atorvastatin than rosuvastatin group $(14.21 \%$ vs $4.38 \%, p<0.05$, respectively). Statin associated muscle symptoms (more severe muscle problem, myositis/myopathy) observed more in atorvastatin than that of rosuvastatin group (34.07\% vs $13.08 \%, p$ $<0.05$, respectively). Both treatments were well tolerated. No cases of rhabdomyolysis, incident diabetes, hepatic or renal insufficiency were recorded during the study period.

Conclusion: Rosuvastatin had better outcome profile of muscle symptoms than atorvastatin in patients with clinical atherosclerotic cardiovascular disease among the Bangladeshi population. Patients in atorvastatin group experienced more muscle pain, fatigue, cramp/spasticity and weakness than rosuvastatin.
\end{abstract}

University Heart Journal 2018; 14(1): 9-20

Introduction:

Atherosclerotic cardiovascular disease (ASCVD) is one of the leading causes of death worldwide. Statin therapy is the cornerstone for prevention and treatment of cardiovascular diseases (CVDs), and is generally safe and well tolerated. ${ }^{1}$ According to ASCVD Primary Prevention Guideline 2016, atherosclerotic cardiovascular diseases refer to the following conditions: a) Coronary heart disease (CHD), such as myocardial infarction (MI), angina, and coronary artery stenosis $>50 \%$. b) 
Cerebrovascular disease, such as transient ischemic attack (TIA), ischemic stroke, and carotid artery stenosis $>50 \%$. c) Peripheral artery disease, such as claudication. d) Aortic atherosclerotic disease, such as abdominal aortic aneurysm (AAA) and descending thoracic aneurysm. Clinical atherosclerotic cardiovascular disease includes acute coronary syndromes, history of myocardial infarction (MI), stable or unstable angina, coronary or other arterial revascularization, stroke, transient ischemic attack or peripheral arterial disease presumed to be of atherosclerotic origin. ${ }^{2}$

The pathophysiological mechanisms of ASCVDs have brought new insight regarding potential indicators of underlying hidden atherosclerosis and cardiovascular risk. ${ }^{3}$ Platelet is the main culprit for thrombus formation in disrupted plaque by which atherosclerosis leads to the acute ischemic syndromes. ${ }^{4}$ High dose statin seems to be beneficial in early phase of thrombosis due to its antiplatelets effects. Study recommended that the management of CVDs includes statin therapy in highrisk conditions including clinical atherosclerosis to decrease the risk of CVD events and mortality. ${ }^{5}$ Hydroxymethyl-glutaryl (HMG) Coenzyme A (CoA) reductase inhibitors or statins are the most effective medications for managing elevated concentrations of LDL-C as well as reduce cardiac events in coronary artery disease (CAD) patients. ${ }^{6}$ The 2013 American College of Cardiology/American Heart Association (ACC/AHA) guideline recommends importance of starting statins with at least moderate-intensity therapy (e.g., simvastatin 40 $\mathrm{mg}$ or atorvastatin $20 \mathrm{mg}$ ). Sometimes, high-intensity statin therapy is also recommended (atorvastatin 40-80 $\mathrm{mg}$ or rosuvastatin $20-40 \mathrm{mg}$ ). The ACC/AHA calculator estimates 10-year risk of "hard ASCVD" (stroke, MI), and it also recommends statins at 7.5\% (0.75\%/year). [7, ${ }^{2]}$ However, the dose and intensity of statin use in ASCVD is debatable among cardiologist. ${ }^{8}$ As our understanding of LDL-C and atherosclerosis continues to grow, the concept of 'lower is better' has corresponded with a 'more is better' approach to statin-based therapy. ${ }^{9}$ There is a close relationship between early atherosclerotic lesions and inflammation which can elicit acute coronary syndromes. ${ }^{[10]}$ Early initiation of high dose statin can limit further progression of inflammation by its pleiotropic effect which is not directly dependent on reduced cholesterol levels. ${ }^{11,12,13}$

Myopathy is separated into three different types: a) myalgia refers to generalized pain in the muscles, associated with muscle damage with small increase of the creatine kinase enzyme. b) Myositis presents itself with muscle pain, tenderness or weakness and a higher level of creatine kinase in the bloodstream. c) Rhabdomyolysis is an extreme, life-threatening type of myopathy. It's brought on by muscle breakdown and significant creatine kinase elevation, up to 40 times greater than normal value. Rhabdomyolysis can be fatal due to acute renal failure. Myalgia can occur with or without creatine kinase (CK) elevation; a serum marker of muscle damage. ${ }^{6}$ The frequency of muscle-related symptoms was the most frequent adverse effects of statins. In community cohorts, it is more than $10 \%$ to $20 \%$ of patients. These adverse effects are usually benign and resolve with treatment interruption but often lead to reluctance to resume statin treatment. Rhabdomyolysis is a severe adverse effect related to statins. ${ }^{14}$

The pharmaceutical company reported the incidence of myalgia during therapy with the more powerful statins has varied from $1 \%$ to $25 \%{ }^{15,16}$ The Prediction of Muscular Risk in Observational Conditions (PRIMO) project has confirmed the clinicians' suspicions of muscle symptoms of statin among 7924 patients treated with high dose statins, $11 \%$ developed muscle symptoms, $4 \%$ had symptoms severe enough to interfere with daily activities, and $0.4 \%$ was actually confined to bed with their symptoms. ${ }^{17}$ The risk for myopathy among statin users was relatively more than controls in cohort and meta analysis. ${ }^{18}$ Muscle symptoms could interfere with an individual's activities of daily living and quality of life and these adverse outcomes are likely to become a cause for poor compliance. Furthermore, statin associated muscle symptoms (SAMS) could interfere with the tolerability of exercise, depriving the individual's cardiovascular benefits of regular exercise. So, present study was conducted to determine the association of muscle symptoms of atorvastatin versus (vs) rosuvastatin in patients with clinical atherosclerotic cardiovascular disease at a tertiary centre of Bangladesh.

\section{Material and Methods:}

\section{Trial design}

This was a 3-month, single blind, randomized, parallel group study conducted in Department of Cardiology, University Cardiac Center, Bangabandhu Sheikh Mujib Medical University, Shahbag, Dhaka, Bangladesh between August 2016 and July 2017. Patients with clinical atherosclerotic cardiovascular disease and/or having dyslipidaemia (LDL-C e" $190 \mathrm{mg} / \mathrm{dl}$ ) were enrolled for study. Then the patient grouped randomly by computer based online random number table. One group was given 
atorvastatin $40 \mathrm{mg}$ and another group rosuvastatin $20 \mathrm{mg}$. In the pharmacologic point of view, atorvastatin $40 \mathrm{mg}$ is equal to rosuvastatin $20 \mathrm{mg}$ and regarded as high intensity statin therapy according to 2013 ACC/AHA guideline. Atorvastatin $80 \mathrm{mg}$ and rosuvastatin $40 \mathrm{mg}$ is recommended as high intensity statin therapy for western population. As the pattern of environment, genetic status and body habitus is different in our country, the lowest doses of high intensive statin therapy was taken as standard. Detailed history and examination including evaluation of associated risk factors was done on first and as well as subsequent visits. Subjects who met the study definition of myalgia (primary outcome) includes all of the following: (1) New or increased muscle pain, cramps, aching not associated with exercise, (2) Symptoms persisted for at least 2 weeks, (3) Symptoms resolved within 2 weeks of stopping the study drug, and (4) symptoms reoccurred within 4 weeks of restarting the study medication. Age (age groups: 20-39, 40-59 and $>60$ years) and sex (male or female) matched were categorized. Subject who tested positive for myalgia with these criteria subsequently was performed serological tests (serum creatine kinase) immediately after occurrence of muscle symptoms. Approval of the study was seeking permission by the local Ethical Committee. All procedures of human subjects were performed in accordance with the latest version of Helsinki Declaration. All subjects were included in the study group were sign upon informed consent with careful explanation of the study procedures.

\section{Patients}

Men and women aged 20 to 74 years of age with clinical atherosclerotic cardiovascular diseases were enrolled consecutively following the inclusion and exclusion criteria. Detailed history taking, physical examination and routine lab examinations were done on admission and outpatient consultation subsequently whenever feasible. Inclusion criteria included: Patients with clinical ASCVD includes-acute coronary syndromes (Acute myocardial infarction, unstable angina, prior history of MI, stable angina, coronary artery revascularization, strokes or transient ischemic attack (TIA), peripheral arterial diseases (PAD). Exclusion criteria included: Age $>75$ or $<20$ years, patients with heart failure with NYHA IIIV, patients with hepatic impairments (ALT value $>2$ times of ULN), patients with renal impairments (Serum creatinine level $>2 \mathrm{mg} / \mathrm{dl}$ ), patients with haemorrhagic strokes, patients with primary muscle disorders, patients with current or prior steroid therapy, patients with rheumatological disorders, patients with hypothyroidism, patients with drugs that alter statin metabolism-diuretics, amiodarone, azole antifungals, macrolides, protease inhibitors, immunosuppressive, prior history of statin hypersensitivity, pregnancy, cancer or prior history of cancer, patient taking statin other than atorvastatin or rosuvastatin, and patients who are not interested to take part in this study were excluded.

\section{Objective}

The primary endpoint was the muscle symptoms of atorvastatin and rosuvastatin at 4 weeks and in 3 months of study period included: muscle pain, muscle stiffness and cramp, weakness and fatigue. Secondary efficacy endpoints included: the demographic variation, frequency, impacts on everyday life of these symptoms. Adverse events and clinical chemistry data with atorvastatin 40 $\mathrm{mg}$ and rosuvastatin $20 \mathrm{mg}$ were also assessed.

\section{Follow-up of study subjects}

The protocol was required that all selected patients were observed at 4 weeks and at 3 months. During visits surveillance for primary endpoint events, medical compliance (pill counting method), adverse events (WHO adverse drug reporting form) and vital status were evaluated. Patients were conducted on regular basis by telephone. If any problem arose in between follow up schedule he advised to contact us or visit local registered physicians or hospital. Data was collected over telephone or by direct surveillance. The primary efficacy end point was muscular effects. In the presence of muscle symptoms, serum creatine kinase was measured to confirm the muscle damage and myalgia and categorized according to ESC definition. Secondary efficacy endpoint was effect on lipid profile, gastrointestinal symptoms, incident diabetes, neurocognitive impairment, peripheral neuropathy, liver enzyme elevation. A standardized questionnaire and examination was used to screen muscle symptoms and potential clinical events during follow up. Adherence to medication and lifestyle, therapeutic response to statin therapy, and safety was regularly assessed.

\section{Treatment protocol}

Atorvastatin $40 \mathrm{mg}$ daily in one group and rosuvastatin $20 \mathrm{mg}$ daily in another group was given to index population. Concomitant use of aspirin, clopidogrel or 
ticagrelor or prasugrel, beta blocker, angiotensins converting enzyme inhibitors (ACEIs) or angiotensin receptor blockers (ARBs), nitrates, antidiabetic medication were permitted in both groups. Drugs that can affect the metabolism of statin like macrolides, amiodarone, diuretics, azole antifungal, protease inhibitors, and immunosuppressive drugs-cyclosporine were prohibited.

\section{Instruction after giving statins}

Patient was instructed about adherence of drugs regularly. Heart healthy diet, avoidance of tobacco products, regular exercise and maintenance of healthy weight (BMI) also advised. Diet and drugs that interact with statins metabolism were also be avoided and were reported after above scheduled or at any adverse situation. The motivated person was enrolled. Positive reinforcement to adhere to treatment will be provided. Telephone survey was done to assess patient's compliance about treatment frequently. All patients were advised to contact immediately if any need arises. Drug interaction and comorbid conditions were assessed exclusively during the full study period. Good knowledge about the other drugs that the patient was taken was updated regularly by using manufacturer papers and as well as from internet.

\section{Assessment of muscle symptoms}

The muscular effects of statins were assessed by the following methods-muscle pain and tenderness by universal pain assessment tool (scale-0 to 10), muscle fatigue by fatigue assessment scale and brief fatigue inventory chart ( scale-1 to 10), muscle cramp/spasticity by modified Ashworth scale (scale-0 to 5), and muscle weakness by MRC muscle power scale ( scale- 0 to 5 ).

\section{Study parameters and evaluation}

All the clinical and biochemical parameters were recorded accurately \& precisely for each patient at baseline, at 4 weeks, 03 months and thereafter, whenever feasible. Clinical parameter included: muscle pain, muscle cramp and stiffness/spasticity, muscle weakness and fatigue. Biochemical parameter included: serum fasting lipid profile (fasting lipid profile is preferable), serum creatine kinase (CK) (selected cases), and serum alanine aminotransferase (ALT) selected cases and urine for myoglobinuria (in selected cases where $\mathrm{CK}>10$ times of ULN).

\section{Outcome variables}

Primary efficacy end point variables

Muscle pain, cramp, stiffness/spasticity, fatigue and weakness

\section{Primary safely end point variables}

Liver enzyme elevation (ALT)

Gastro-intestinal discomfort (abdominal pain, nausea, vomiting)

\section{Peripheral neuropathy (tingling, numbness)}

Neurocognitive symptoms: Insomnia, Memory impairment

Incident diabetes

\section{Statistical analysis}

Statistical analyses were planned and reviewed by the scientific committee. Analysis was conducted on SPSS windows software including multiple imputations for missing data. Primary analysis was carried out on intentto- treat basis. Descriptive statistical analysis for each of the parameters assessed was compared in patients with and without muscular symptoms on the basis of mean values and standard deviations (continuous variables) or percentages (categorical variables). Univariate analysis was use to identify factors associated with increased risk of muscular symptoms and odds ratio (OR) with $95 \%$ two sided confidence intervals (CI) will be calculated. Continuous parameter was express as mean $\pm \mathrm{SD}$ and categorical parameters is express as median and inters quartile range. Pearson's Chi-square test for categorical variables and student's t-test for continuous variables were used to compare two groups. Multivariate logistic regression was performed to calculate the adjusted ORs with $95 \%$ CIs to estimate the correlation between risk factors and muscular symptoms. The significance thresholds in two-tailed tests were taken as 0.05. Time of onset of muscular symptoms after statin therapy were $\log$-transformed to verify that time of onset were unimodal. Outcomes were assessed for normality by use of normal probability plots and histograms

\section{Results:}

This single blind randomized clinical controlled trial was conducted in the University Cardiac Center, Bangabandhu Sheikh Mujib Medical University, Dhaka, over a period of one year from August 2016 to July 2017. Finally, a total 280 patients were selected for the study at 1:1 randomization by computer generated random number table. No patient was missed to follow up as sample size was limited. Two visits were designed. First visit was at 4 weeks and subsequent last visit was in 3 months of treatment period. Age of the study population was ranging from 20 to 74 years. It was observed that the majority 172 patients $(61.43 \%)$ belonged to age $40-59$ years. The mean age was found $53.3 \pm 10.9$ years in atorvastatin group 
and $53.7 \pm 11.6$ years in rosuvastatin group. Male patients were 245 and female were 35 . Sex difference between two groups was statistically not significant as sex match was done between two groups. Body mass index (mean \pm SD) $25 \pm 6.5$ for male patients and $24 \pm 5.5$ for female patients in atorvastatin group and $25 \pm 6.8$ for male patients and $24 \pm 5.4$ for female patients in rosuvastatin group. There was no statistical significant variation of BMI between groups. The distribution of the patients according to indication for statin therapy showed common indication was non ST elevated myocardial infarction (NSTEMI), $28.57 \%$ and $27.14 \%$ in atorvastatin and rosuvastatin group respectively. Chronic stable angina (CSA) was $20 \%$ vs $18.57 \%$ in atorvastatin and rosuvastatin group respectively. Indication for statin therapy between two groups was no significant difference.

All patients were instructed about the Wong-Baker facial grimace scale in which $0=$ no pain and $10=$ severe pain. Score $\geq 3$ denotes moderate to severe pain. Patients in atorvastatin groups experienced more pain than rosuvastatin. In atorvastatin group $3.57 \%$ had moderate pain, $1.43 \%$ had severe muscle pain and $19.29 \%$ had mild pain at 4 weeks. In rosuvastatin group only $2.14 \%$ had moderate pain and $10.72 \%$ had mild pain. No patient had severe pain in rosuvastatin group in 4 weeks duration. Two (2) patients had to discontinue atorvastatin and five (5) patients had to require dose reduction due to muscle pain in atorvastatin group. Difference of muscle pain between two groups was statistically significant $(\mathrm{p}<0$ $.05)$. Subsequently in 3 months of the study period, $34.59 \%$ patients had mild pain, $15.04 \%$ had moderate pain and $12.78 \%$ had severe muscle pain in atorvastatin group. In rosuvastatin group only $4.38 \%$ had severe muscle pain, $6.57 \%$ had moderate pain and $27 \%$ patients had mild pain. Prior 9 patient of mild pain subsequently developed moderate and 5 patients developed severe pain in atorvastatin group. In rosuvastatin group, prior 3 patients of mild pain developed moderate and 2 patients developed severe pain subsequently in 3 months of treatment duration. Difference of muscle pain between two groups was statistically significant $(\mathrm{p}<0.05$.

In our study, clinical improvements were subjectively assessed by the patient's statement of his/herself feelings or thought regarding symptoms by fatigue assessment scale. A significant number of patients of both groups felt bad (2.86\% vs $3.57 \%$ respectively) at 4 weeks and felt extremely bad ( $12.78 \%$ vs $4.37 \%$ respectively) or bad ( $21.80 \%$ vs $10.95 \%$ respectively) in 3 months of the study period. The difference was statistically also significant at $\mathrm{p}<0.05$.
In this study, muscle spasticity grading showed that, patients in atorvastatin group observed substantial increase in muscle spasm than rosuvastatin. In atorvastatin group, 15 patients observed slight increase muscle spasm and 1 patient more marked increase muscle spasm whereas 3 patients showed slight increase muscle spasm in rosuvastatin group at 4 weeks duration. In atorvastatin group, one (1) patient showed considerable increase, five (5) more marked increase and 34 patients showed slight increase muscle spasm in 3 months of study period. In rosuvastatin group, only 2 patients had more marked increase and 19 patients showed slight increase muscle spasm in 3 months of statins therapy. The difference was statistically also significant at $\mathrm{p}<0.05$.

On evaluation of medical research council (MRC) muscle power scale, maximum patients (95\% vs $97.86 \%$ respectively) showed grade 5 (normal power) at 4 weeks of study period whereas $2.26 \%$ patients had MRC grade 3 (can move limb against gravity) in atorvastatin group and $15.03 \%$ vs $8.76 \%$ patients showed MRC grade 4 between atorvastatin and rosuvastatin group respectively. No patient in rosuvastatin group showed 3 of less MRC scale grade during the study period. The difference was statistically also significant at $\mathrm{p}<0.05$.

Adverse profile of medication showed that, patients in atorvastatin groups observed liver enzyme elevation $(\mathrm{ALT})<2$ times of ULN was $3.57 \%$, but it was $1.42 \%$ in rosuvastatin group. In atorvastatin group, $8.57 \%$ of patients developed gastrointestinal symptoms (pain, nausea) and $5 \%$ in rosuvastatin group. No other significant adverse event developed in both groups at 4 weeks of treatment period. Further development of adverse profile of medication showed that, patients in atorvastatin groups observed liver enzyme elevation (ALT) 2-3 times of ULN was $9.77 \%$ and it was $8.03 \%$ in rosuvastatin. In atorvastatin group, $3.76 \%$ of patients developed gastrointestinal symptoms (pain, nausea) and $5.11 \%$ in rosuvastatin group. $1.42 \%$ patients developed peripheral neuropathy in both groups. No other significant adverse event developed in both groups in 3 months of treatment period.

In atorvastatin group, $19.15 \%$ patients and in rosuvastatin group, $10.72 \%$ had creatine kinase $(\mathrm{CK})>$ (upper limit normal) ULN - <4x ULN. 4.28\% patients in atorvastatin group and $2.14 \%$ in rosuvastatin group had CK $>4 \mathrm{x}$ ULN - $<10 x$ ULN. In atorvastatin group, $1.42 \%$ patients had CK $>10 \times$ ULN. Subsequently in 3 months treatment period, in atorvastatin group, $34.33 \%$ patients and in rosuvastatin group, $27 \%$ had $\mathrm{CK}>\mathrm{ULN}-<4 \mathrm{x}$ ULN. In 
atorvastatin group, $15.67 \%$ patients and in rosuvastatin group $6.57 \%$ had $\mathrm{CK}>4 \mathrm{x}$ ULN - <10x ULN. In atorvastatin group, $12.69 \%$ patients and in rosuvastatin group $4.38 \%$ had $\mathrm{CK}>10 \mathrm{x}$ ULN. One (1) patient of atorvastatin group of spasticity grade 4 showed CK $>4 \mathrm{x}$ ULN. Difference of serum creatine kinase (CK) between two groups was statistically significant $(\mathrm{p}<0.05)$.

The clinical presentation of muscle symptoms was highly heterogeneous, as reflected by the variety of manifestation like muscle pain or aching, stiffness, tenderness or cramp. In this study, severe muscle problem and myopathy or myositis referred to statin associated muscle symptoms (SAMS). As normal population, patients with simple myalgia showed some degree of increased CK level ( $<4$ times of ULN). The present study showed that total $42(15 \%)$ patients of statin therapy referred as myalgia, in which 27 patients were in atorvastatin group and 15 patients in rosuvastatin group at 4 weeks of treatment period. In atorvastatin group, 6 patients developed severe muscle problem and 2 developed myositis or myopathy. In rosuvastatin group,
3 patients developed severe muscle problem and none developed myositis or myopathy.

Total $83(61.03 \%)$ patients of statin therapy referred as myalgia, in which 46 patients were in atorvastatin group and 37 patients were in rosuvastatin group in 3 months of the study period. In atorvastatin group, 21 patients developed severe muscle problem and 17 developed myositis or myopathy. In rosuvastatin group, 9 patients developed severe muscle problem and 6 patients developed myositis or myopathy. Difference of statinassociated muscle symptoms (SAMS) between two groups was statistically significant $(\mathrm{p}<0 \quad .05)$.

Study showed that the frequency of muscle symptoms was more in atorvastatin group than that of rosuvastatin group. Statin induced muscle symptoms (severe muscle problem and myopathy or myositis) in atorvastatin was 8 $(5.71 \%)$ and $3(2.14 \%)$ in rosuvastatin at 4 weeks of treatment period. Statin induced muscle symptoms (severe muscle problem and myopathy or myositis / SAMS) in atorvastatin was $38(28.35 \%)$ and $15(10.94 \%)$ in rosuvastatin group in 3 months period of treatment.

Table-I

Patients demographics and baseline characteristics (randomized population)

\begin{tabular}{lcc}
\hline Characteristics & $\begin{array}{c}\text { Atorvastatin } 40 \mathrm{mg} \\
(\mathrm{n}=140)\end{array}$ & $\begin{array}{c}\text { Rosuvastatin } 20 \mathrm{mg} \\
(\mathrm{n}=140)\end{array}$ \\
\hline Mean age, years (SD) & $53.3 \pm 10.9$ & $53.7 \pm 11.6$ \\
Male gender, $\mathrm{n}(\%)$ & 85.71 & 89.28 \\
BMI, kg/m ${ }^{2}$ (SD) & & \\
Male & $25 \pm 6.5$ & $25 \pm 6.8$ \\
Female & $24 \pm 5.5$ & $24 \pm 5.4$ \\
Diabetes, $\mathrm{n}(\%)$ & 25 & 27.14 \\
Hypertension, $\mathrm{n}(\%)$ & 30.71 & 32.45 \\
Co-medications ${ }^{\text {a }}$, Other than statins, n (\%) & 99.28 & 99.28 \\
& & \\
Indication of statin therapy, n (\%) & 10 & 11.42 \\
Acute ST elevated myocardial infarction (STEMI) & 28.57 & 27.14 \\
Non ST elevated myocardial infarction (NSTEMI) & 12.14 & 13.57 \\
Unstable angina & 20 & 18.57 \\
Stable angina & 9.2 & 12.14 \\
Coronary artery revascularization & 16.42 & 12.14 \\
Prior history of MI & 1.42 & 0.71 \\
Strokes or TIA & 2.14 & 2.85 \\
Peripheral arterial diseases (PAD) & 12.85 & 10.71 \\
Smoking & 1.42 & 2.14 \\
Alcohol & & \\
\hline
\end{tabular}

SD, standard deviation; BMI, body mass index.

Data were expressed as percentage and mean $\pm \mathrm{SD}$, percentage values are expressed as percentage of the total number of patients in each group.

${ }^{\mathrm{a}} \mathrm{Co}$-medications-beta-blocker, angiotensin receptor blocker, angiotensin converting enzyme inhibitor, anxiolytics, antidepressant, antidiabetic medication 
Table-II

Character and different scale and grade of muscle symptoms between drugs

\begin{tabular}{|c|c|c|c|c|c|c|c|}
\hline \multirow{2}{*}{\multicolumn{2}{|c|}{$\begin{array}{l}\text { Characteristics(muscle symptoms } \\
\text { grades/scales) }\end{array}$}} & \multicolumn{2}{|c|}{ At 4 weeks } & \multirow[t]{2}{*}{ P-value } & \multicolumn{2}{|c|}{ In 3 months } & \multirow[t]{2}{*}{ P-value } \\
\hline & & $\begin{array}{c}\text { Atorvastatin } \\
(\mathrm{n}=140)\end{array}$ & $\begin{array}{l}\text { Rosuvastatin } \\
\quad(\mathrm{n}=140)\end{array}$ & & $\begin{array}{c}\text { Atorvastatin } \\
\quad(\mathrm{n}=133)\end{array}$ & $\begin{array}{l}\text { Rosuvastatin } \\
\quad(\mathrm{n}=137)\end{array}$ & \\
\hline \multicolumn{8}{|c|}{ Wong-Baker Facial grimace scale } \\
\hline 0 & $106(75.71)$ & $122(87.14)$ & & $50(37.59)$ & $85(62.05)$ & & \\
\hline $1-2$ & $27(19.29)$ & $15(10.72)$ & .00344 & $46(34.59)$ & $37(27)$ & .00014 & \\
\hline $3-6$ & $5(3.57)$ & $3(2.14)$ & & $20(15.04)$ & $9(6.57)$ & & \\
\hline $7-10$ & $2(1.43)$ & $0(0)$ & & $17(12.78)$ & $6(4.38)$ & & \\
\hline \multicolumn{8}{|c|}{ Fatigue scale } \\
\hline & Feeling extremely bad & 0 & 0 & & $17(12.78)$ & $6(4.37)$ & \\
\hline & Feeling bad & $4(2.86)$ & $5(3.57)$ & 0.523 & $29(21.80)$ & $15(10.95)$ & .00035 \\
\hline & Feeling neutral & $50(35.71)$ & $51(36.43)$ & & $35(26.32)$ & $40(29.20)$ & \\
\hline & Feeling good & $25(17.86)$ & $25(17.86)$ & & $40(30.07)$ & $58(42.34)$ & \\
\hline & Feeling extremely good & $61(43.57)$ & $59(42.14)$ & & $12(9.02)$ & $18(13.14)$ & \\
\hline \multicolumn{8}{|c|}{ Spasticity grade } \\
\hline & No increase in muscle spasm/spasticity & $124(88.57)$ & $137(97.86)$ & & $93(69.93)$ & $116(84.67)$ & \\
\hline & No increase in muscle spasm/spasticity & $15(10.71)$ & $3(2.14)$ & .0084 & $34(25.56)$ & $19(13.87)$ & .00044 \\
\hline & No increase in muscle spasm/spasticity & $1(0.71)$ & 0 & & $5(3.76)$ & $2(1.46)$ & \\
\hline & No increase in muscle spasm/spasticity & 0 & 0 & & $1(0.75)$ & 0 & \\
\hline & No increase in muscle spasm/spasticity & 0 & 0 & & 0 & 0 & \\
\hline \multicolumn{8}{|c|}{ MRC scale } \\
\hline & 0 & 0 & 0 & & 0 & 0 & \\
\hline & 1 & 0 & 0 & & 0 & 0 & \\
\hline & 2 & 0 & 0 & & 0 & 0 & \\
\hline & 3 & 0 & 0 & & $3(2.26)$ & 0 & \\
\hline & 4 & $7(5)$ & $3(2.14)$ & .0692 & $20(15.03)$ & $12(8.76)$ & .0092 \\
\hline & 5 & $133(95)$ & $137(97.86)$ & & $110(82.71)$ & $125(91.24)$ & \\
\hline \multicolumn{8}{|c|}{ Statin Associated Muscle Symptoms (SAMS) } \\
\hline & Severe muscle problem & $6(4.29)$ & $3(2.14)$ & .00041 & $21(15.67)$ & $9(6.56)$ & .00001 \\
\hline & Myositis/myopathy & $2(1.42)$ & 0 & & $17(12.69)$ & $6(4.38)$ & \\
\hline
\end{tabular}

Data were expressed as frequency and percentage, Chi-square test was used to see the association between groups, $\mathrm{N}=$ number of the study population, ns=not significant

Table-III

Clinical description of muscular symptoms during study period $(N=168)$

\begin{tabular}{|c|c|c|c|c|c|c|}
\hline \multirow[t]{2}{*}{ Characteristics } & \multirow[t]{2}{*}{ Description } & \multicolumn{2}{|c|}{ Atorvastatinn $_{1}=103$} & \multicolumn{2}{|c|}{ Rosuvastatinn $_{2}=65$} & \multirow[t]{2}{*}{ P-value } \\
\hline & & $\mathrm{N}$ & $\%$ & $\mathrm{~N}$ & $\%$ & \\
\hline \multirow[t]{2}{*}{ Widespread pain } & Yes & 31 & 30.09 & 23 & 35.38 & $.024^{\mathrm{ns}}$ \\
\hline & No & 72 & 69.91 & 42 & 64.61 & $.074 \mathrm{~ns}$ \\
\hline \multirow[t]{7}{*}{ Site of pain } & Thigh/calves & 25 & 25.24 & 18 & 27.69 & $.081^{\mathrm{ns}}$ \\
\hline & All over & 31 & 30.09 & 23 & 35.38 & $.347 \mathrm{~ns}$ \\
\hline & Trunk & 13 & 12.62 & 7 & 10.76 & $.061 \mathrm{~ns}$ \\
\hline & Arm/forearm & 8 & 7.77 & 5 & 7.69 & $.078^{\mathrm{ns}}$ \\
\hline & Tendonitis associated pain & 22 & 21.35 & 10 & 15.38 & $.095 \mathrm{~ns}$ \\
\hline & No predominant site & 4 & 3.88 & 2 & 3.07 & $.457^{\mathrm{ns}}$ \\
\hline & Unknown & 0 & 0 & 0 & 0 & 0 \\
\hline
\end{tabular}

Data were expressed as frequency and percentage, Chi-square test was used to see the association between groups, $\mathrm{N}=$ number of the study population, ns $=$ not significant 
Table-IV

Frequency, impact on everyday life and management of muscular symptoms $(N=168)$

\begin{tabular}{|c|c|c|c|c|c|c|}
\hline \multirow[t]{2}{*}{ Variables } & & \multicolumn{2}{|c|}{$\begin{array}{l}\text { Atorvastatin } \\
\left(\mathrm{n}_{1}=103\right)\end{array}$} & \multicolumn{2}{|c|}{$\begin{array}{l}\text { Rosuvastatin } \\
\left(\mathrm{n}_{2}=65\right)\end{array}$} & \multirow[t]{2}{*}{ P-value } \\
\hline & & No. & $\%$ & No. & $\%$ & \\
\hline \multirow[t]{2}{*}{ Frequency of pain } & Continuous & 19 & 18.5 & 6 & 9.2 & $.001^{\mathrm{s}}$ \\
\hline & Intermittent & 84 & 81.5 & 59 & 90.8 & $.0831^{\mathrm{ns}}$ \\
\hline \multirow{2}{*}{$\begin{array}{l}\text { Pain requiring symptomatic } \\
\text { treatment }\end{array}$} & No & 59 & 57.3 & 47 & 72.3 & \\
\hline & Yes & 44 & 42.7 & 18 & 27.7 & $.002^{\mathrm{s}}$ \\
\hline \multirow[t]{4}{*}{ Level of disruption of daily life } & Minor disruption & 39 & 37.8 & 30 & 46.1 & $.321^{\mathrm{ns}}$ \\
\hline & Interfere with major exertion & 20 & 19.4 & 17 & 26.1 & $.0056^{\mathrm{s}}$ \\
\hline & Interfere with moderate exertion & 25 & 24.3 & 12 & 18.5 & $.735 \mathrm{~ns}$ \\
\hline & Major disruption & 19 & 18.5 & 6 & 9.3 & $.031^{\mathrm{s}}$ \\
\hline \multirow{2}{*}{$\begin{array}{l}\text { Clinical management of } \\
\text { symptoms }\end{array}$} & No & 59 & 57.3 & 47 & 72.3 & $.853 \mathrm{~ns}$ \\
\hline & Yes & 44 & 42.7 & 18 & 27.7 & $.006^{\mathrm{s}}$ \\
\hline \multirow{2}{*}{$\begin{array}{l}\text { Discontinuation of } \\
\text { statin }\end{array}$} & No & 84 & 81.5 & 59 & 90.8 & \\
\hline & Yes & 19 & 18.5 & 6 & 9.2 & $.001^{\mathrm{s}}$ \\
\hline \multirow{2}{*}{$\begin{array}{l}\text { Reduction in dosage of } \\
\text { statin }\end{array}$} & No & 78 & 75.7 & 53 & 81.5 & \\
\hline & Yes & 25 & 24.3 & 12 & 18.5 & $.0021^{\mathrm{s}}$ \\
\hline
\end{tabular}

\section{Discussion:}

Although a number of previous studies have compared atorvastatin with rosuvastatin in patients with hypercholesterolemia, no one included muscle problems of high intensity statin among the Bangladeshi population. It was a single blind randomized controlled trial (RCT) where 280 patients of clinical atherosclerotic cardiovascular disease were studied to compare high intensity statins; atorvastatin $40 \mathrm{mg}$ with rosuvastatin 20 mg. Muscle pain and tenderness, fatigue, cramp and spasticity and weakness were studied and in symptomatic patients creatine kinase and alaline aminotransferase were investigated. The results of the study demonstrated that rosuvastatin was better than atorvastatin in all aspects of the outcome variables.

Muscular pains (aches) and cramps were the earlier manifestation of muscle symptoms than fatigue and weakness. About 103 patients in atorvastatin and 65 in rosuvastatin group experienced some degree of muscle pain (Table II) during the study period. Patients with severe pain had to discontinue statins. Patients with moderate pain had to reduce the doses of statins in both groups as well.

Both localized and widespread pain (Table III) was noticed (69.91\% versus/vs $30.09 \%$ and $64.62 \%$ vs 35.38 in atorvastatin and rosuvastatin respectively). Localized pain was affecting trunk, thighs and calves, arms and hands. A significant number of patients $(21.31 \%$ vs $15.38 \%$ in atorvastatin and rosuvastatin respectively) also experienced tendon related pain (tendonitis). Frequency, impact on everyday life and management of muscular symptoms (Table IV) after statin therapy showed that continued pain was $18.5 \%$ vs $9.2 \%$, intermittent pain was $81.5 \%$ vs $90.8 \%$ in atorvastatin and rosuvastatin group respectively. Pain required symptomatic treatment was $42.7 \%$ vs. $18 \%$, major disruption of daily life was $18.5 \%$ vs. $9.3 \%$, patients had to discontinue statins were $18.5 \%$ vs. 9.2 and $24.3 \%$ vs $18.5 \%$ patients had to dose reduction of atorvastatin and rosuvastatin respectively.

In our study, clinical improvements were subjectively assessed by the patient's statement of his/herself feelings or thought regarding symptoms by fatigue assessment scale. A significant number of patients of both groups felt bad ( $2.86 \%$ vs $3.57 \%$ respectively) at 4 weeks and felt extremely bad $(12.78 \%$ vs $4.37 \%$ respectively) or bad ( $21.80 \%$ vs $10.95 \%$ respectively) in 3 months of the study period (Table II). The difference was statistically also significant at $\mathrm{p}<0.05$.

In this study, muscle spasticity grading showed that, patients in atorvastatin group observed substantial increase in muscle spasm than rosuvastatin. In atorvastatin group, 15 patients observed slight increase muscle spasm and 1 patient more marked increase muscle spasm whereas 3 patients showed slight increase muscle spasm in rosuvastatin group at 4 weeks duration. In atorvastatin group, one (1) patient showed considerable increase, five 
(5) more marked increase and 34 patients showed slight increase muscle spasm in 3 months of study period. In rosuvastatin group, only 2 patients had more marked increase and 19 patients showed slight increase muscle spasm in 3 months of statins therapy (Table II).

On evaluation of medical research council (MRC) muscle power scale, maximum patients (95\% vs $97.86 \%$ respectively) showed grade 5 (normal power) at 4 weeks of study period whereas $2.26 \%$ patients had MRC grade 3 (can move limb against gravity) in atorvastatin group and $15.03 \%$ vs $8.76 \%$ patients showed MRC grade 4 between atorvastatin and rosuvastatin group respectively. No patient in rosuvastatin group showed 3 of less MRC scale grade during the study period (Table II).

Symptomatic patients tended to be more active than the population as a whole. Index patient selection, physical activities, drugs, infection, smoking, diabetes, hypertension, alcohol, elevated liver enzyme, gastrointestinal symptoms, peripheral neuropathy were found to be non independent predictors of the study outcome by univariate and multivariate regression analysis. Age, gender and body mass index were not risk factors for muscular symptoms. Creatine kinase (CK) level elevation associated with significant impact on symptomatic patients in both groups both in 4 weeks and 3 months of treatment period. Patients with severe muscle problems and myositis or myopathy with $\mathrm{CK}$ level $>4$ times of ULN referred as statin associated muscle symptoms (SAMS).

In the absence of a standardized classification of SAMS, European Atherosclerosis Society Consensus Panel propose to integrate all muscle-related complaints (e.g. pain, weakness, or cramps) as 'muscle symptoms', subdivided by the presence of CK elevation. ${ }^{[19]}$ Creatine kinase is the enzyme released from damaged muscle cells, and $\mathrm{CK}$ elevations $>10 \times$ the upper limit of normal (ULN) occur in 1 per 1000 to 1 per 10000 people per year, depending on the statin, its dose, and the presence of other risk factors. ${ }^{20}$

The majority of the patients belonged to age 40-59 years. The mean age was found $53.3 \pm 10.9$ years in atorvastatin group and $53.7 \pm 11.6$ years in rosuvastatin group. Male patients were 245 and female were 35 and body mass index tended to be overweight. Male patients were substantially more in number because more male patients were admitted or attended in the department of cardiology of BSMMU and were enrolled subsequently. It was not statistically significant as because sex match was uniformly distributed between two groups of the study population.

This finding is almost similar to the other study. In a study age group ranging between 30 to 80 years with a mean age of 58.21 years where the highest incidence was seen between 41 and 50 years with male to female ratio of $4: 1$ in acute coronary syndromes. [3] Pain and weakness in typical SAMS are usually symmetrical and proximal, and generally affect large muscle groups including the thighs, buttocks, calves, and back muscles and occur early (within 4-6 weeks after starting statin therapy but may still occur after many years of treatment. [6] The symptoms appear to be more frequent in physically active individuals. ${ }^{17}$

On evaluation of clinical and laboratory findings, present study showed that the frequency of muscle symptoms were more in atorvastatin group. A total 42 patients at 4 weeks and 83 patients in 3 months of statin therapy referred as 'myalgia' with normal CK level, in which 73 patients were in atorvastatin and 52 patients were in rosuvastatin group. In atorvastatin group, statin induced muscle symptoms (severe muscle problem, myositis or myopathy / SAMS) were $8(5.71 \%)$ and $3(2.41 \%)$ in rosuvastatin group at 4 weeks and $38(28.57 \%)$ vs 15 $(10.94 \%)$ in atorvastatin and rosuvastatin group respectively in 3 months of the study period.

Findings of our study accordance with result of other study. Patient registries, together with clinical experience, indicate that $7-29 \%$ of patients complain of statin-associated muscle symptoms (SAMS). ${ }^{17,21,22,}$ 23,24 These are usually associated with elevated CK concentrations. Statin induced muscle symptoms in atorvastatin is $14.9 \%$ (PRIMO study, 2006) and $7.6 \%$ in rosuvastatin (Jupiter trial, 2008).

Result of our study consistent with statement of other study. Muscle symptoms range from myalgia, which includes muscle pain without creatine kinase (CK) elevations, to myositis which is muscle symptoms with CK elevations. ${ }^{[25]}$ In general, elevations of CK of more than ten times the upper limit of normal are regarded as significant elevations justifying the discontinuation of statin treatment. ${ }^{26}$ The majority of patients who complain of muscle symptoms have mild/moderately elevated $\mathrm{CK}$ levels $(<4 \times \mathrm{ULN}){ }^{27}$

Both atorvastatin and rosuvastatin were well tolerated. Most adverse events were of mild or moderate severity. 
Liver enzyme elevation (e.g. alaline aminotransferase), gastrointestinal symptoms (e.g. abdominal pain, nausea), peripheral neuropathy (e.g. tingling, numbness) were reported in both groups with similar incidence. No patient of incident diabetes or rhabdomyolysis was found during the study period.

Our study design raised a number of important methodological issues, including patient selection, follow-up, sample size and the prospective evaluation of muscle problems of the statin therapy, all of which exerted a powerful influence on the results. Withdrawal of statin therapy followed by one or more re-challenges (after a washout) can often help in determining causality; additional approaches include the use of an alternative statin, a statin at lowest dose, intermittent (i.e. non-daily) dosing of a highly efficacious statin, or the use of otherlipid lowering medications. For patients at low cardiovascular (CVD) risk, their need for a statin should be reassessed and the benefits of therapeutic lifestyle changes, such as cessation of cigarette smoking, blood pressure control and dietary modification should be balanced against the risk of continuing statin therapy. Conversely, patients at high CVD risk, including those with CVD or diabetes mellitus, the benefits of ongoing statin therapy need to be weighed against the burden of muscle symptoms.

\section{Conclusion:}

Statin-associated muscle symptoms (SAMS) frequently cause statin non-adherence, switching and discontinuation, contributing to adverse cardiovascular (CV) outcomes. In this study, statin induced muscle symptoms (severe muscle problem and myositis/ myopathy / SAMS) was $34.07 \%$ in atorvastatin group and $13.08 \%$ in rosuvastatin. A definitive diagnosis of SAMS is difficult because symptoms are subjective and there is no gold standard diagnostic test. A clinical diagnosis was made on the basis of a patient's medical history, the clinical presentation and the association of the patient's symptoms (muscle pain, stiffness or cramps, fatigue and weakness) with statin therapy over time. So, proper evaluation and early detection can reduce the burden of SAMS.

\section{Limitation}

\section{Recommendations}

- Ensure that there is an indication for statin use and that the patient is fully aware of the expected benefit in cardiovascular disease risk reduction that can be achieved with this treatment.

- Counsel patients regarding the risk of 'side effects' and the high probability that these can be dealt with successfully.

- Periodic CK as well as others markers of muscle injury and inflammation (e.g. serum myoglobin, lactate dehydrogenase, hs C-reactive protein) measurement.

- A large scale study with various doses with different statins can be conducted in future to reach to a definitive conclusion

This study was not without limitations. The limitations of the study were as follows:

- Study was conducted amongst patients with clinical atherosclerotic cardiovascular disease on generally, but not clarified type, pattern or severity of CVD.

- It was a single centre study. Only patients admitted in Department of Cardiology, BSMMU were taken for the study. So this will not reflect the overall picture of the country.

- Muscle symptoms were studied in fixed doses of high intensity statin therapy of atorvastatin and rosuvastatin only.

- Small sample size and single serum CK measurement in symptomatic patients instead of periodic and generalized measurement.

- Lipid profiles, liver function tests, renal function tests were not done routinely done during study period.

- Male patients were substantially high.

\section{Acknowledgements:}

I would like to acknowledge the enthusiastic guidance of my beloved teacher and guide Prof. Dr. Harisul Hoque, Professor, Clinical \& Interventional Cardiologist, Dept. of Cardiology, BSMMU, Dhaka. I express my heartfelt gratitude to him in choosing the topic and title, close supervision, guidance and encouragement in every step of this study, without which this work would not have been possible. It is an immense pleasure to have such dedicated and helpful co-guides Dr. Jahanara Arzu and Dr. Dipal Krishna Adhikary, Associate Professors, Dept. of Cardiology, BSMMU, Dhaka to recognize the problems, constant inspiration, information and support 
provided by them. It is my great pleasure and privilege to express my deep gratitude to Prof. Dr. Sajal Krishna Banerjee, Professor and Chairman, Prof. Dr. Syed Ali Ahsan, Professor and Head of Interventional Cardiology, Prof. Dr. Chowdhury Meshkat Ahmed Prof. Dr AKM Fazlur Rahman, Prof. Dr. Mohammad Safiuddin and Prof. Dr. SM Mustafa Zaman, Department of Cardiology, BSMMU, Dhaka, for their invaluable scientific suggestions, helpful criticism, support, co-operation , inspiration, valuable advices in thesis writing and continued motivation throughout the research period. My sincere regards and gratefulness to Dr. Khorshed Ahmed, Dr. Md. Mukhlesur Rahman and Dr. Manzoor Mahmood, Associate Professors, Dept. of Cardiology, BSMMU, for their constant source of stimulations and suggestions in performing the work. I convey my profound gratitude and indebtedness to Dr. Golam H Rabbani, Department of Public Health \& Informatics, BSMMU for his intense co-operation and guidance in the study procedure all over including the sample size determination. I am thankful to all of my family members, all consultants, medical officers, and my coursemets of BSMMU, Dhaka, for their co-operation and constant motivation. It will be unfair, if I do not express my gratitude to all the patients, who were the subjects of this study. My sincere thanks them for being of my study subject.

\section{References:}

1. Reiner Z, Catapano AL, De Backer G, Graham I, Taskinen MR, Wiklund O, Agewall S, Alegria E, Chapman MJ, Durrington P, Erdine S, Halcox J, Hobbs R, Kjekshus J, Filardi PP, Riccardi G, Storey RF, Wood D. ESC/EAS guidelines for the management of dyslipidaemias: the Task Force for the management of dyslipidaemias of the European Society of Cardiology (ESC) and the European Atherosclerosis Society (EAS). Eur Heart J, 2011;32:1769-18.

2. ACCF/AHA Pocket Guideline. Management of Patients With Unstable Angina/ Non-ST-Elevation Myocardial Infarction. $J$ Am Coll Cardiol 2011;57: 215-367.

3. Hemalatha N, Siddanathi N, Devi K, Chandrakala B, and Krishna G. A study on Role of circulating hsCRP as a biomarker of clinical significance in acute Myocardial infarction (MI) and identification of extent of involvement, risk assessment and post MI complications. Journal of Basic Medical and Allied Sciences (JBMAS) 2015;4:192-99.

4. Fuster V, Badimon L, Badimon J, Chesebro JH. The pathogenesis of coronary artery disease and the acute coronary syndromes. $N$ Engl JMed 1992;326:242-50.

5. Anderson T, Gregoire G, Pearson G, et al. Canadian Cardiovascular Society Guidelines for the Management of Dyslipidemia for the
Prevention of Cardiovascular Disease in the Adult. Canadian Journal of Cardiology 2016;32 (2016):1263-1282.

6. Parker BA, Capizzi JA, Grimaldi AS, Clarkson PM, Cole SM, Keadle J, Chipkin S, Pescatello LS, Simpson K, White CM, Thompson PD. Effect of statins on skeletal muscle function. Circulation 2013;127:96-103.

7. Stone N, Robinson G, Lichtenstein A, et al. 2013 ACC/AHA Guideline on the Treatment of Blood Cholesterol to Reduce Atherosclerotic Cardiovascular Risk in Adults- A Report of the American College of Cardiology/American Heart Association Task Force on Practice Guidelines. Journal of the American College of Cardiology 2014; 63(25): 2891-2931.

8. Yan, Qin Qin, Songbai Deng, Ling Wu, Yajie Liu, Xiaodong Jing, Jianlin Du, Qiang She. Benefit and risk comparison between highand moderate-intensity statin therapy in patients with atherosclerotic cardiovascular disease: a mixed treatment comparison meta-analysis. PROSPERO 2015:CRD42015024753

9. Kapur N, Musunuru K. Clinical efficacy and safety of statins in managing cardiovascular risk. Vascular Health and Risk Management. 2008;4(2):341-353.

10. Hansson G. Inflammation, Atherosclerosis, and Coronary Artery Disease. N Engl J Med 2005;352:1685-95.

11. Takemoto M, Liao JK. Pleiotropic effects of 3-hydroxy-3methylglutaryl coenzyme A reductase inhibitors. Arterioscler Thromb Vasc Biol 2001;21:1712-9.

12. Crisby M, Nordin-Fredriksson G, Shah PK, Yano J, Zhu J, Nilsson J. Pravastatin treatment increases collagen content and decreases lipid content, inflammation, metalloproteinases, and cell death in human carotid plaques: implications for plaque stabilization. Circulation 2001;103:926-33.

13. Sposito AC, Chapman MJ. Statin therapy in acute coronary syndromes: mechanistic insight into clinical benefit. Arterioscler Thromb Vasc Biol 2002;22:1524-34.

14. Downs J, and Patrick G. O'Malley. Management of Dyslipidemia for Cardiovascular Disease Risk Reduction: Synopsis of the 2014 U.S. Department of Veterans Affairs and U.S. Department of Defense Clinical Practice Guideline. Ann Intern Med. 2015;163:291-297.

15. Physicians' Desk Reference. Montvale, NJ: Medical Economics; 2002.p. 56.

16. Phillips PS, Haas RH, Bannykh S, Hathaway S, Gray NL, Kimura BJ, Vladutiu GD, England JD. Statin-associated myopathy with normal creatine kinase levels. Ann Intern Med. 2002; 137(7): 581-5.

17. Bruckert E, Hayem G, Dejager S, Yau C, Begaud B. Mild to moderate muscular symptoms with high-dosage statin therapy in hyperlipidemic patients - the PRIMO study. Cardiovasc Drugs Ther 2005;19:403-414

18. Macedo AF, Taylor FC, Casas JP et al. Unintended effects of statins from observational studies in the general population: systematic review and meta-analysis. BMC Med 2014; 12: 51.

19. Stroes S, Thompson P, Corsini A, Vladutiu G, Raal F, Ray K, et al. Society Consensus Panel Statement on Assessment, Aetiology and Management. European Heart Journal (2015), 36:1012-1022.

20. Law M, Rudnicka AR. Statin safety: a systematic review. Am J Cardiol 2006;97: 52C-60C. 
21. Buettner C, Rippberger MJ, Smith JK, Leveille SG, Davis RB, Mittleman MA, et al. Statin use and musculoskeletal pain among adults with and without arthritis. Am JMed, 2012;125:176-182.

22. Cohen JD, Brinton EA, Ito MK, Jacobson TA. Understanding statin use in America and gaps in patient education (USAGE): an internetbased survey of 10,138 current and former statin users. J Clin Lipidol, 2012;6:208-15.

23. El-Salem K, Ababeneh B, Rudnicki S, Malkawi A, Alrefai A, Khader $\mathrm{Y}$, et al. Prevalence and risk factors of muscle complications secondary to statins. Muscle Nerve, 2011; 44:877-881.

24. Zhang H, Plutzky J, Skentzos S, Morrison F, Mar P, Shubina M. et al. Discontinuation of statins in routine care settings: a cohort study. Ann Int Med, 2013;158;526-34.
25. Vasudevan A, Y. S. Hamirani, and P. H. Jones. "Safety of statins: effects on muscle and the liver," Cleveland Clinic Journal of Medicine, 2005;72(11):990-1001.

26. Barakat L, Jayyousi A, Bener A, et al. Comparison of Efficacy and Safety of Rosuvastatin, Atorvastatin and Pravastatin among Dyslipidemic Diabetic Patients. Hindawi Publishing Corporation, ISRN Pharmacology, Volume 2013, Article ID 146579, 7 pages

27. Alfirevic A, Neely D, Armitage J, Chinoy H, Cooper RG, Laaksonen R, Carr DF, Bloch KM, Fahy J, Hanson A, Yue QY, Wadelius M, Maitland-van Der Zee AH, Voora D, Psaty BM, Palmer CN, Pirmohamed M. Phenotype standardization for statin-induced myotoxicity. Clin Pharmacol Ther 2014;96:470-476. 